This document is the accepted manuscript version of the following article:

Gaiser, S., Schütz, U., \& Hegemann, D. (2019). Top-down approach to attach liquid polyethylene glycol to solid surfaces by plasma interaction. Plasma Processes and Polymers, e1900211 (7 pp.). https://doi.org/10.1002/ppap.201900211

Plasma Process. Polym. 2019; e1900211; https://doi.org/10.1002/ppap.201900211 Article type: Communication

\title{
Top-Down Approach to Attach Liquid Polyethylene Glycol (PEG) to Solid Surfaces by Plasma Interaction
}

Sandra Gaiser*, Urs Schütz, Dirk Hegemann

S. Gaiser, Urs Schütz, D. Hegemann

Empa, Swiss Federal Laboratories for Materials Science and Technology, Plasma \& Coating Group, Lerchenfeldstrasse 5, 9014 St. Gallen, Switzerland

E-mail: sandra.gaiser@empa.ch

A novel top-down approach is presented to produce stable functional films from low vapor pressure liquids via plasma processing. Low-pressure plasma activation and deposition are examined to cross-link liquid polyethylene glycol (PEG-400) surfaces. After stabilizing the thus obtained films on the liquid with a silicone backing layer, water contact angle (WCA) measurements, Fourier-transform infrared (FTIR) spectroscopy and scanning electron microscope (SEM) imaging were used for characterization. The results show that PEG molecules are covalently bond to the solid films providing high hydrophilicity. 


\section{Introduction}

Depositing solid materials on liquids is a promising approach in many fields and can be conducted by using a variety of methods, substrates and precursors. ${ }^{[1]}$ Low-pressure deposition processes have successfully been used to deposit metallic, polymeric and siliconelike films onto liquids. ${ }^{[1-9]}$ These methods show promising potential for various applications like the encapsulation of liquids, the production of micro-structures and the fabrication of membranes. $^{[1,7,8,10]}$

Low-pressure plasma processes are of particular interest in the preparation of thin films on low vapor pressure liquids since UV radiation, radicals and energy-rich ions lead to an additional cross-linking of molecules in the liquid substrate or between liquid and solid coating. As we will show in the following, not only freely floating thin films can be deposited in this way but they may also be equipped with specific surface features originating from liquid molecules that are attached to the film. We selected liquid polyethylene glycol (PEG) as a substrate material since it features a low vapor pressure and is known for its high hydrophilicity and related non-fouling properties, which are especially crucial for the fabrication of biomedical devices. ${ }^{[11-14]}$

Various methods are available for the immobilization of PEG on solid surfaces. ${ }^{[11,13]}$ However, most of them require multiple pre-treatment steps, including the immersion of samples in different solvents and monomers. ${ }^{[15-17]}$ While this is time-consuming, costly and harmful to the environment, the PEG coating is often not stable, especially on surfaces which are exposed to aqueous solutions since PEG molecules are lost over time due to their good solubility. ${ }^{[18-20]}$ Plasma technology is thus a preferable alternative, which has been used to immobilize and cross-link liquids like PEG and to graft them on top of solid substrates. ${ }^{[15,21]}$ 
However, the molecular structure of PEG gets lost due to fragmentation processes occurring in the plasma. ${ }^{[13,20]}$

To bond PEG molecules to a surface without completely losing their characteristic structure, responsible for the superb hydrophilic and non-fouling properties, we propose to use a plasma process in a top-down approach: thin plasma-polymerized films are directly formed on the surface of liquid substrates. The aim is to covalently bond molecular chains of liquid PEG to the solid film, which is growing on top of the liquid and prevents further cross-linking by shielding it from the plasma. With this novel approach, the formation of a solid-liquid vertical gradient might be achieved, in which the solid surface is equipped with partly fixed but still mobile molecular chains. Furthermore, we present a method to stabilize the sample by adding a silicone backing layer, where the plasma-generated layer additionally serves as a barrier between silicone and PEG. After curing the silicone, the liquid side of the sample can be turned upside down and is used for further surface analysis via water contact angle (WCA) measurements, Fourier-transform infrared (FTIR) spectroscopy and scanning electron microscope (SEM) imaging.

\section{Experimental Section}

For the plasma treatment of liquids a symmetric, capacitively coupled plasma reactor was used operating at low pressure conditions. Polyethylene glycol (PEG-400, Sigma-Aldrich) was selected as a liquid substrate. The individual process steps for the plasma treatment and stabilization of the samples are depicted in Figure 1. Thin layers of liquid PEG were filled in petri dishes, which were then placed in the plasma reactor.

Different plasma processes were chosen to investigate the effect of plasma treatment and plasma deposition processes on the cross-linking and immobilization of PEG molecules. In a first experiment, Ar plasma at a pressure of 0.1 mbar with a gas flow rate of $40 \mathrm{sccm}$ and a power input of $50 \mathrm{~W}$ was applied for a duration of $10 \mathrm{~min}$. For the second run, $\mathrm{O}_{2}$ with a gas 
flow rate of 10 sccm was added to the Ar flow keeping otherwise the same plasma parameters. A third process consisted of a pre-treatment with Ar plasma as described above followed by a coating process to deposit an amorphous hydrocarbon (a-C:H) film using $16 \mathrm{sccm}$ ethylene $\left(\mathrm{C}_{2} \mathrm{H}_{4}\right)$ with a power input of $30 \mathrm{~W}$. The pressure was initially set at $0.2 \mathrm{mbar}$ for $1 \mathrm{~min}$ and was then reduced to $0.1 \mathrm{mbar}$ for another $9 \mathrm{~min}$. The higher pressure conditions provided mild deposition conditions with low ion energies at the liquid-solid interface, followed by enhanced cross-linking during film growth by lowering pressure. ${ }^{[22]}$ For the resulting a-C:H films, a thickness of about $200 \mathrm{~nm}$ was determined.

The surfaces produced in this way were covered with a 2 mm thick layer of liquid polydimethylsiloxane (PDMS) mixed with the corresponding cross-linking agent (Dowsil/Sylgard 184 Silicone Elastomer Kit). By curing the silicone for several hours, the films were stabilized after plasma treatment. The samples were then removed from the petri dishes and immersed into demineralized water for rinsing. The water-soluble liquid PEG, which had not been attached to the plasma-generated film, was largely washed away leaving covalently bonded PEG at the surface of the silicone backing layer. 
plasma
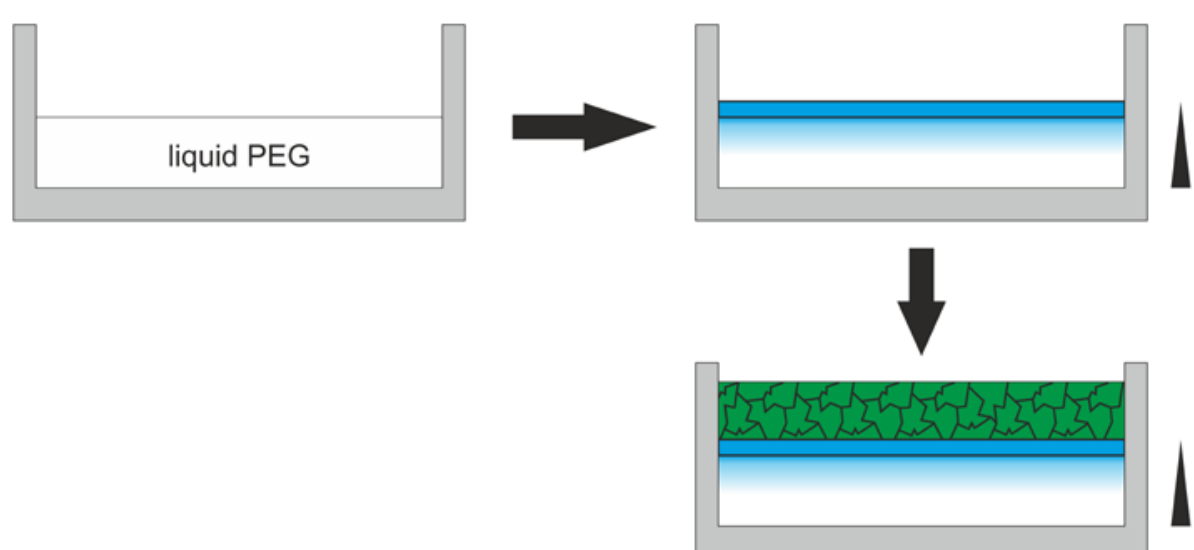

solid plasma coating

covalently bonded liquid

liquid PEG

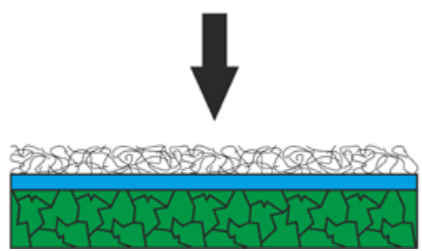

PDMS with crosslinker

solid plasma coating

covalently bonded liquid

liquid PEG

covalently bonded PEG plasma-polymerized film solid PDMS

Figure 1. Schematic of the sample preparation process. Liquid PEG, held in a petri dish, is treated/coated by a plasma. Molecular chains are either bond to a layer of cross-linked PEG or to an a-C:H film. To stabilize the plasma-processed sample, it is covered with liquid PDMS. After curing, the sample can be removed from the petri dish. After rinsing, only covalently bonded PEG is left at its surface.

To characterize the samples measurements of the apparent water contact angle (WCA) were conducted using a Drop Shape Analyzer DSA25. Each mean value reported in this work was calculated using the results of twelve measurements. WCA measurements were repeated after washing the samples a second time. FTIR spectra were taken with a Varian 640-IR spectrometer in ATR mode. For SEM imaging a Hitachi S-4800 device was used. To prepare the samples for the analysis via SEM a plasma coated PEG sample was additionally sputtercoated with a $40 \mathrm{~nm}$ thick gold-platinum film before adding the liquid PDMS. After curing and washing as described before the samples were cut to obtain a cross section through the 
layer system. The contrast between metal coating, silicone and a-C:H film helped to distinguish the different layers by SEM.

\section{$3 \quad$ Results and Discussion}

\subsection{Optical Appearance of the Samples}

The "PEG sides" of the plasma treated and coated samples were first visually inspected. Photographs of the samples are displayed in Figure 2 (a). In comparison to pure silicone and untreated PEG, which feature a completely transparent and shiny appearance, the surfaces of the samples prepared using Ar plasma activation of liquid PEG showed merely a slight blur. The plasma treatment with $\mathrm{Ar}$ and $\mathrm{O}_{2}$ resulted in surfaces showing patches that appear slightly matt. The combination of Ar plasma and subsequent a-C:H plasma coating led to a strong, uniform matt effect on the entire sample surface.

The change in appearance of the samples can be explained by the different plasma processes applied. The Ar plasma treatment can be assumed to cause a certain degree of cross-linking of PEG molecules at the liquid's surface leading to the weakly visible blurring. The increasing visibility of the surface effect from $\mathrm{Ar}$ to $\mathrm{Ar} / \mathrm{O}_{2}$ plasma treatment indicates an increasing degree of cross-linking and attachment of PEG. Thus, these PEG films formed a more stable barrier for the liquid PDMS. The surface showing matt and transparent areas suggests that PDMS was at least partly supported by and attached to the solidified PEG layer. On the samples which were coated by using an $\mathrm{C}_{2} \mathrm{H}_{4}$ plasma, a uniform, non-transparent surface is clearly visible, which can be assigned to the plasma-polymerized a-C:H film. This $200 \mathrm{~nm}$ thick solid layer clearly withstood the process of stabilizing the samples with silicone and subsequent rinsing, since it appeared to be still intact. 
(a)

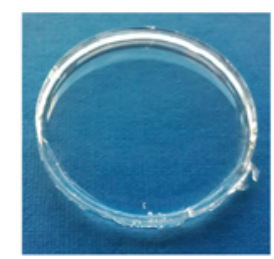

PDMS

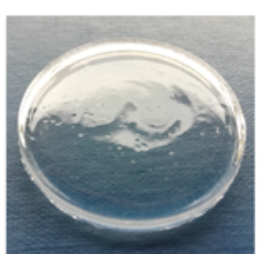

PEG, Ar

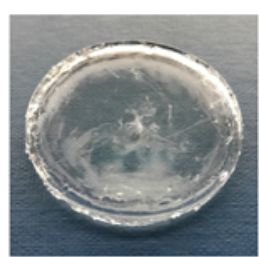

PEG, $\mathrm{Ar}+\mathrm{O}_{2}$

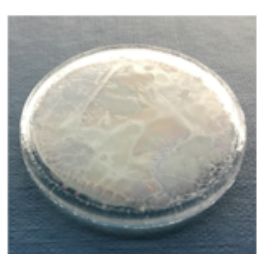

PEG, $\mathrm{Ar}, \mathrm{C}_{2} \mathrm{H}_{4}$

(b)

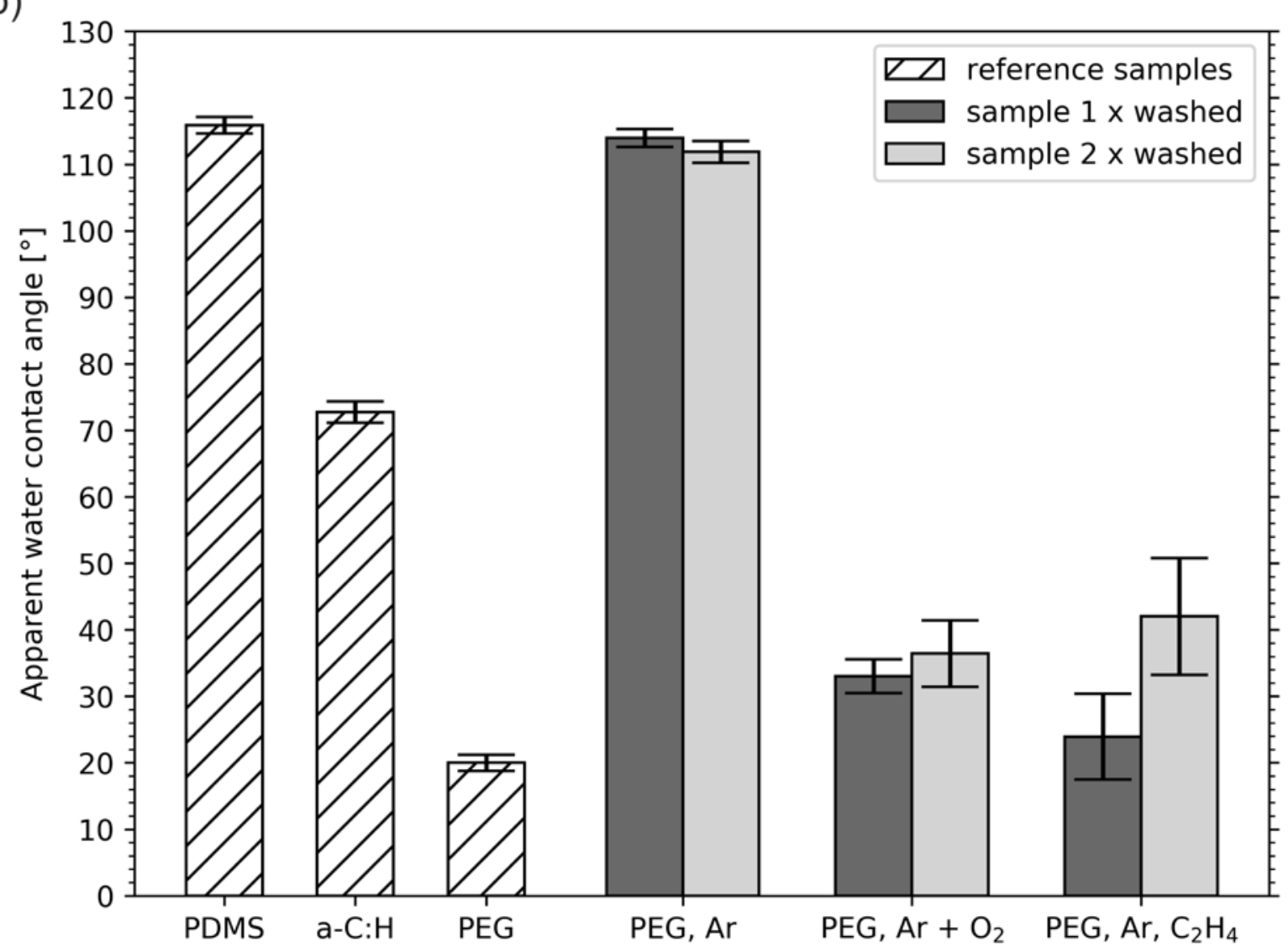

Figure 2. (a) Photographs of pure PDMS and plasma treated PEG samples with PDMS backing. (b) WCA measured on reference samples (cured silicone (PDMS), plasmapolymerized film (a-C:H), plasma treated PEG) and on the plasma treated/coated PEG samples which were stabilized with PDMS and removed from the petri dishes. Values measured after a first and a second rinsing procedure are displayed.

\subsection{Water Contact Angle Measurements}

Since PEG is known to be very hydrophilic, measurements of the apparent water contact angle were conducted to examine the surface properties of the samples and the possibility of 
PEG being attached. First, various reference samples were analyzed. The obtained values are displayed by the hatched bars in Figure 2 (b). The WCA of cured PDMS (116 $)$ as well as of the a-C:H film $\left(73^{\circ}\right)$ were determined. On thin films of liquid PEG treated with an $\mathrm{Ar} / \mathrm{O}_{2}$ plasma for 10 minutes, a WCA of $20^{\circ}$ was measured. Considering a certain error this corresponds to values from literature where minimum contact angles between $23^{\circ}$ and $31^{\circ}$ are reported for immobilized PEG. ${ }^{[23,24]}$

The results of the WCA measurements on the plasma treated/coated samples, which were stabilized with PDMS and removed from the petri dishes, are represented by the filled bars in Figure 2 (b). Dark bars correspond to the first set of measurements after washing the samples once. After repeating the rinsing procedure, WCA were measured again, displayed by the filled, light bars. For the samples treated with Ar plasma, high contact angles between $111^{\circ}$ - $114^{\circ}$ were measured. These values are comparable with the WCA measured on cured PDMS and therefore indicate that no PEG layer is attached to the silicone backing layer.

After washing, the $\mathrm{Ar} / \mathrm{O}_{2}$ plasma treated samples showed low WCA of $33^{\circ}$ in the areas where an optical effect was visible as described before. The transparent spots, however, featured the same values as pure PDMS, again indicating that this plasma treatment did not lead to an uniform surface. This is probably due to a lack in stability of the cross-linked PEG film and a partial subsidence of liquid PDMS into the PEG. The samples coated by $\mathrm{C}_{2} \mathrm{H}_{4}$ plasma showed a WCA of $23^{\circ}$ after the first washing procedure. The uniform optical appearance of the samples correlates well with the equal distribution of low WCA values throughout their surfaces. A comparison with the reference values for the a-C:H film and PEG clearly reveals that the hydrophilic surface properties of the $\mathrm{Ar} / \mathrm{O}_{2}$ plasma treated and the coated samples are caused by attached PEG. The WCA slightly increased to $36^{\circ}$ and $42^{\circ}$, respectively, after rinsing the samples a second time. This might be due to the additional applied mechanical stress slightly damaging the PEG layer or further removal of residual weakly-bond PEG molecules. However, the surfaces remain highly hydrophilic. We could also show that this 
high degree of functionalization can even be maintained for at least several months, superior to optimized functional plasma polymer films. ${ }^{[25]}$ Hence, covalent attachment of liquid molecules to plasma-produced films can be assumed providing them with specific functionalities. Note that the washing procedure might even be avoided or weakened to aim for a pronounced solid-liquid gradient leaving also non-covalently bound PEG molecules at the surface.

\subsection{FTIR Spectroscopy}

To further investigate the presence of PEG at the samples' surfaces, FTIR spectra were recorded. Figure 3 (a) displays the spectra of cured PDMS and liquid PEG-400. The spectra of the plasma treated/coated samples are plotted in Figure 3 (b). Comparison of the spectra allows drawing conclusions about the attachment of PEG to the samples. 
(a)

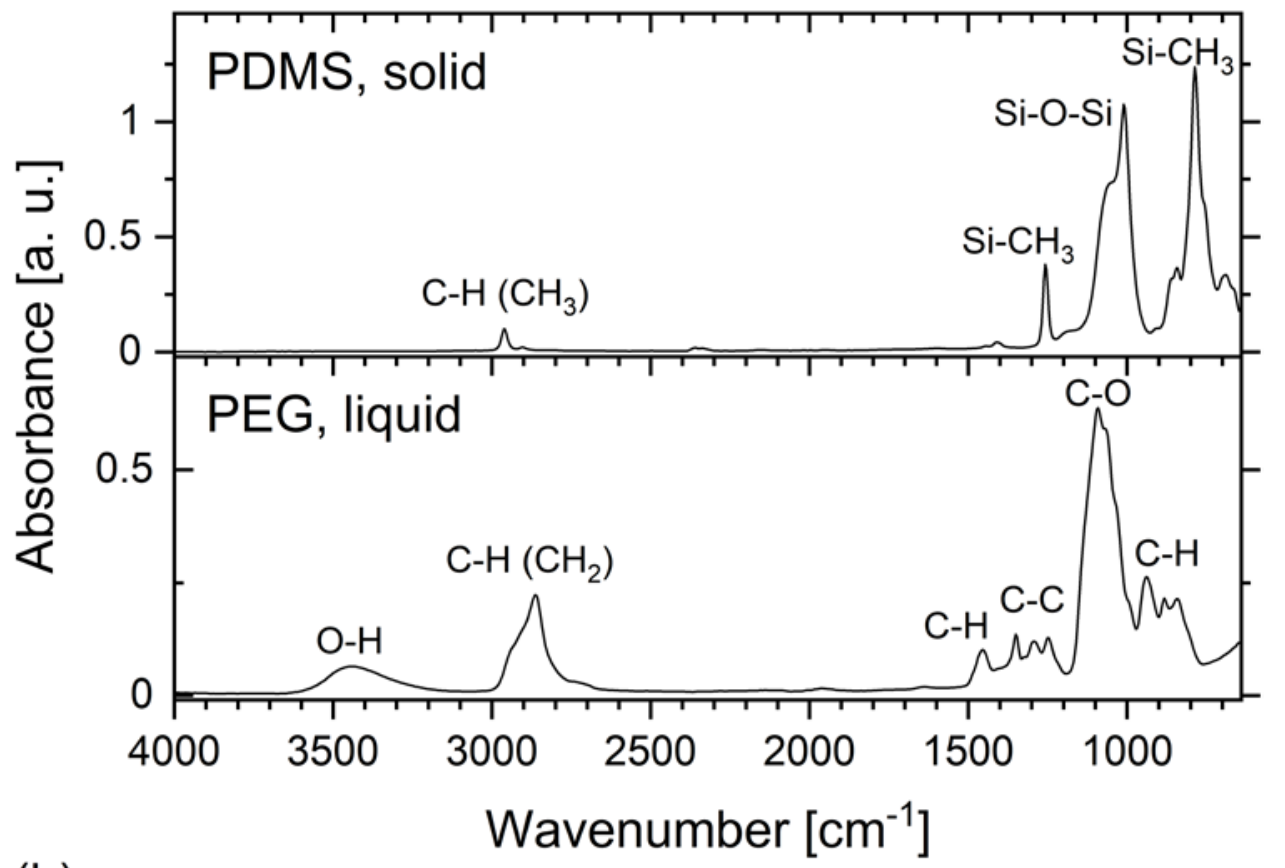

(b)

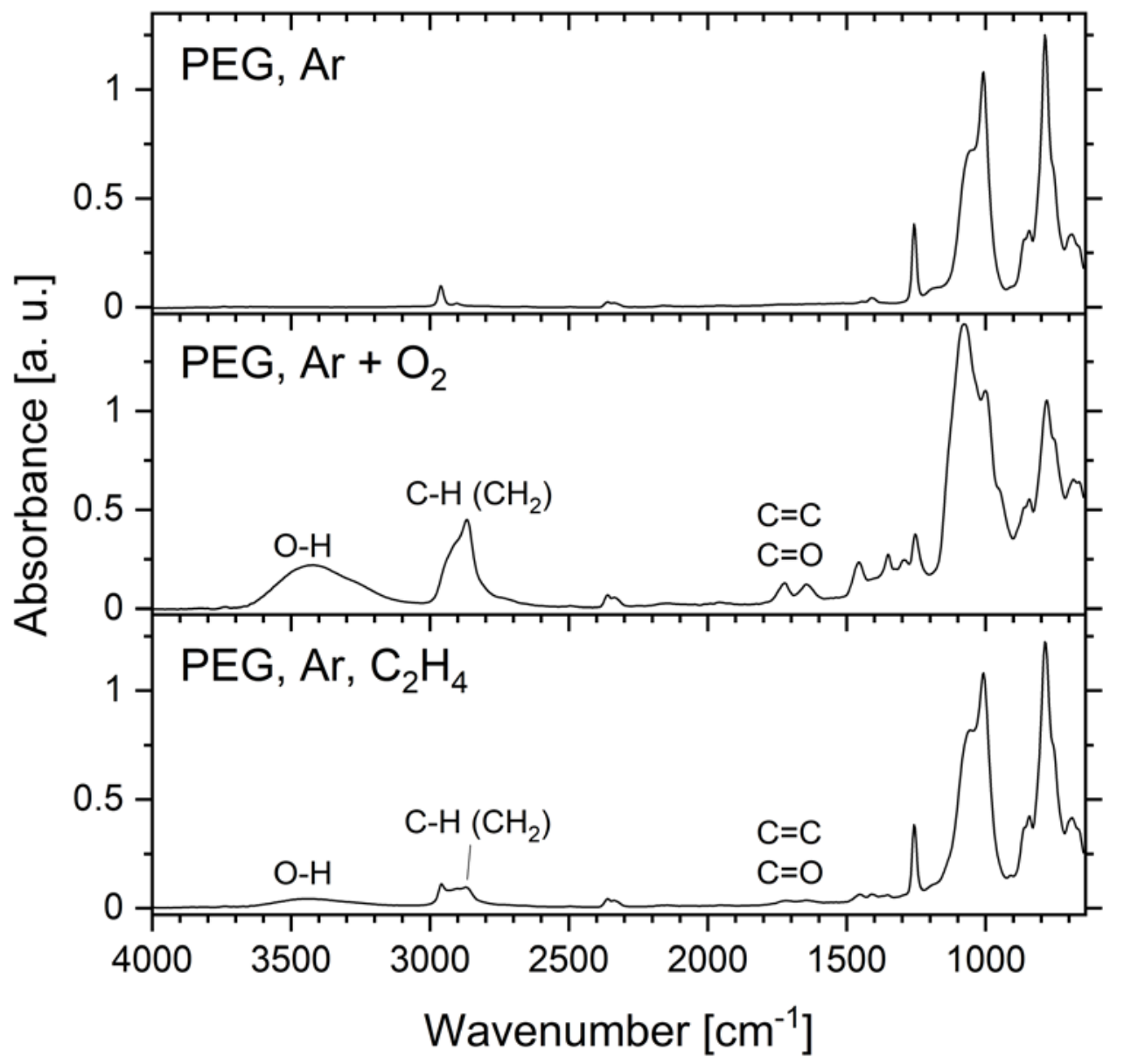

Figure 3. (a) FTIR spectra of cured silicone (PDMS, solid) and liquid PEG-400. (b) FTIR spectra of PEG-400 samples which had been treated by $\mathrm{Ar}$ and $\mathrm{Ar} / \mathrm{O}_{2}$ plasma and coated by $\mathrm{C}_{2} \mathrm{H}_{4}$ plasma, respectively. 
PDMS shows its characteristic absorption bands at $1010 \mathrm{~cm}^{-1}$ and $1060 \mathrm{~cm}^{-1}$. They correspond to the stretching vibration of Si-O-Si. The bands around $1257 \mathrm{~cm}^{-1}$ and $787 \mathrm{~cm}^{-1}$ indicate the $\mathrm{Si}-\mathrm{CH}_{3}$ bonding. Another peak at $2962 \mathrm{~cm}^{-1}$ is caused by the $\mathrm{C}-\mathrm{H}$ bond of $\mathrm{CH}_{3}$. $\mathrm{PEG}$ can easily be distinguished from PDMS as its O-H bond can clearly be identified between $3100 \mathrm{~cm}^{-1}$ and $3650 \mathrm{~cm}^{-1}$. The distinct peak at $2866 \mathrm{~cm}^{-1}$ stems from the $\mathrm{C}-\mathrm{H}$ bond of $\mathrm{CH}_{2}$ and is shifted to lower wave numbers compared with the C-H signal of PDMS since $\mathrm{C}$ and $\mathrm{H}$ appear in different configurations in the two polymers. Characteristic C-C bands appear between $1190 \mathrm{~cm}^{-1}$ and $1420 \mathrm{~cm}^{-1}$ and the $\mathrm{C}-\mathrm{H}$ deformation of $\mathrm{CH}_{2}$ at $1454 \mathrm{~cm}^{-1}$. Further C-H bands can be found between $755 \mathrm{~cm}^{-1}$ and $968 \mathrm{~cm}^{-1}$. The pronounced double band of the C-O stretching vibration appears at $1060 \mathrm{~cm}^{-1}$ and $1092 \mathrm{~cm}^{-1}$, close to the Si-O-Si signal of PDMS. The comparison of the spectrum of the Ar plasma treated sample with PEG and PDMS references did not reveal any difference to PDMS indicating that only the solid PDMS background is present. This finding is in accordance with the optical observations and WCA measurements that did not show a significant visible effect or low WCA values hinting at an attachment of PEG unlike for the other two plasma-processed samples. The $\mathrm{Ar} / \mathrm{O}_{2}$ plasma treated (only matt areas) and plasma coated samples featured differences in their spectra compared to the PDMS spectrum as could be expected considering the distinct change in hydrophilic properties. In fact, the spectra show the characteristic bands of PEG, which are described above, specifically the $\mathrm{O}-\mathrm{H}$ bond and the $\mathrm{C}-\mathrm{H}$ bond at $2866 \mathrm{~cm}^{-1}$. The C-C bands between $1190 \mathrm{~cm}^{-1}$ and $1420 \mathrm{~cm}^{-1}$ and the C-H signal at $1454 \mathrm{~cm}^{-1}$ are also visible, most clearly for the $\mathrm{Ar} / \mathrm{O}_{2}$ plasma treated samples. Compared with the spectra of PDMS the samples showing typical PEG signals also reveal two additional peaks at $1650 \mathrm{~cm}^{-1}$ and $1720 \mathrm{~cm}^{-1}$, which can be assigned to the absorption of $\mathrm{C}=\mathrm{C}$ and $\mathrm{C}=\mathrm{O}$. Their appearance is associated with a partial cross-linking of single PEG molecules. ${ }^{[26]}$ 
The distinctive PEG signature seems to be more pronounced for the samples treated with an $\mathrm{Ar} / \mathrm{O}_{2}$ plasma than for the coated ones. However, it has to be noted that the spectra were normalized to the Si-O-Si peak of the silicone backing at $1010 \mathrm{~cm}^{-1}$. This signal may be weaker for the coated samples since the PDMS layer is further away from the sample's surface due to the intermediate plasma-polymerized layer. Therefore, no direct comparison between intensity values can be made. The signal of the a-C:H film itself can be neglected since it contributes only weak signals of $\mathrm{C}-\mathrm{C}$ and $\mathrm{C}-\mathrm{H}$ bonds, which overlap with the visible absorbance bands of PDMS and PEG.

The spectra proved that PEG was attached to the samples treated with $\mathrm{Ar} / \mathrm{O}_{2}$ plasma, which indicates that a partly cross-linked layer of PEG has been formed. FTIR also clearly confirmed that PEG was bond to the plasma-polymerized a-C:H films.

\subsection{Scanning Electron Microscopy}

The previous results proved that PEG can be attached to samples' surfaces using suitable plasma conditions within the presented top-down approach. In order to obtain further insight into the surface structure, SEM images of the plasma coated samples were recorded looking at the cross section where the sample had been cut. In Figure 4, the thick silicone backing layer is visible in the upper part (left image), which can well be distinguished from the intermediate metal layer that was introduced to increase the contrast in SEM. The further layer system consisting of the metal coating, the a-C:H film and the attached PEG can be identified underneath the silicone. Due to the limited adhesion between the sputtered metal film and the silicone backing, detaching occurred in some areas during sample preparation. Magnification (right image) reveals the layer sequence with known thicknesses, where the brighter film can be assigned to the metal coating with a thickness of $\sim 40 \mathrm{~nm}$. The darker layer underneath corresponds to the plasma-polymerized film with a thickness of $\sim 200 \mathrm{~nm}$. The additional 
layer attached to the plasma coating can thus be identified as adsorbed PEG. Comparing the thickness scale reveals that its thickness is in the same range as the one of the a-C:H film.
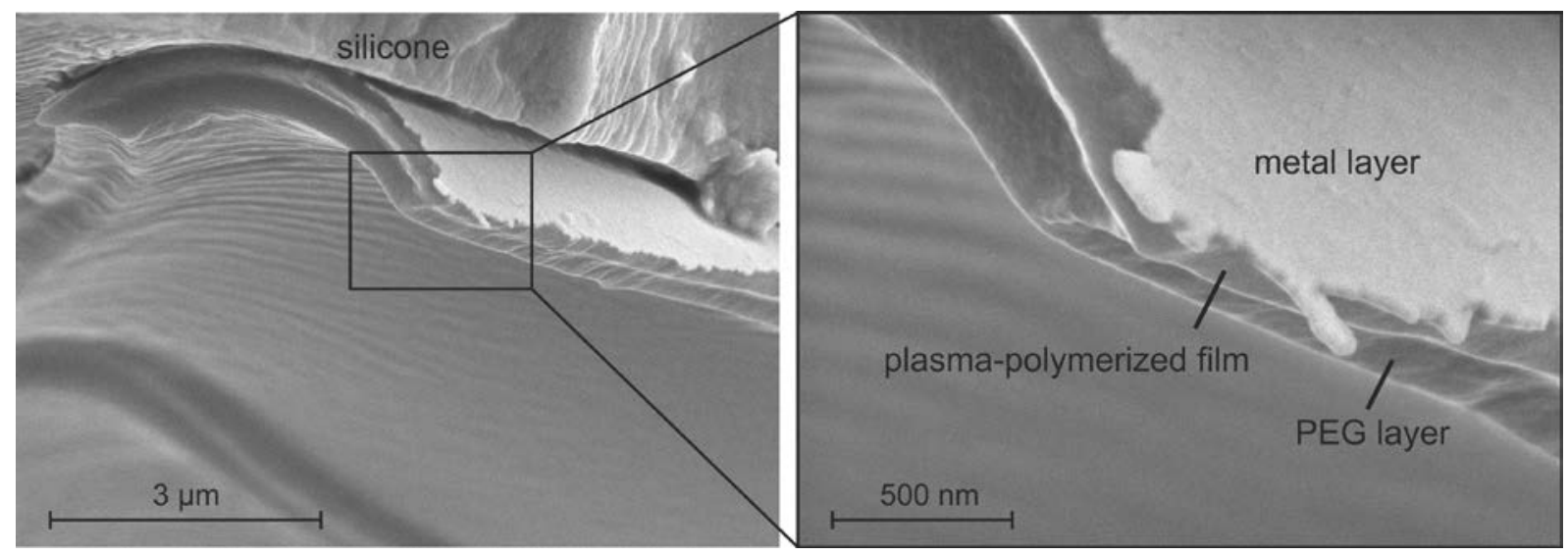

Figure 4. SEM images of the edge of a sample of PEG treated with an Ar plasma and afterwards coated using a $\mathrm{C}_{2} \mathrm{H}_{4}$ plasma. Left: The metal film is partly detached from the silicone. Right: Attached to the metal layer the plasma-polymerized film and a layer of adsorbed PEG are visible.

The length of a single PEG molecule can be determined by considering the length of one PEG monomer molecule $\left(0.35 \mathrm{~nm}^{[27]}\right)$ and the molecular weight of PEG-400, which corresponds to nine repeating units in the molecular chain. This results in a chain length of $\sim 3 \mathrm{~nm}$.

Comparing it with the thickness of the PEG layer in the range of 200 nm indicates the depth of the cross-linking effect during plasma pre-treatment and coating. At the surface of the sample, however, free molecular PEG chains and chain ends might be expected. It will be the subject of subsequent investigations to verify this and to further explore the presented approach.

\section{Conclusion}

A novel top-down approach has been introduced to covalently bond polyethylene glycol to a surface via a low-pressure plasma process. Two different plasma treatment processes and one 
coating process were applied to liquid PEG, held in petri dishes. A method has been developed to stabilize the samples with an additional layer of silicone. It has been demonstrated that the plasma-generated films are able to serve as stable support for the silicone backing and as barrier preventing the silicone from mixing with the liquid PEG. The samples could be neatly removed from the petri dishes and were analyzed visually and by means of WCA measurements, FTIR spectroscopy and SEM imaging. Use of Ar plasma resulted in a surface showing weak evidence of partial cross-linking of PEG. However, no layer was formed which would have withstood the stabilization procedure. Subsequent measurements did not show any signs of PEG being attached to the sample. Applying Ar/O 2 plasma resulted in partly cross-linked PEG layers. Ar plasma treatment followed by $\mathrm{C}_{2} \mathrm{H}_{4}$ plasma lead to closed a-C:H films. Measurements of the apparent contact angle showed a highly hydrophilic character of the samples produced by these two types of plasma processes, even after repeated washing of the samples. This indicates that PEG is not only present at the surface but is also covalently bound to it. Further evidence for a successful attachment of PEG was obtained by recording FTIR spectra that showed typical absorption bands of the PEG molecule. SEM was used to visualize the PEG layer attached to the a-C:H film, which allowed estimating its thickness of $200 \mathrm{~nm}$.

The results clearly show that the presented method is a valuable approach to attach PEG to a solid, plasma-generated film. Furthermore, the introduced top-down approach represents a sound basis for further exploitation, also including different liquid substances used to functionalize surfaces. 
Acknowledgements:

This work was partly funded by the EMPAPOSTDOCS-II program, which has received funding from the European Union’s Horizon 2020 research and innovation program under the Marie Skłodowska-Curie grant agreement No. 754364.

Keywords: immobilization of molecules; liquid substrates; plasma polymerization; plasma treatment; poly(ethylene glycol) (PEG)

References:

[1] A. Homsy, E. Laux, L. Jeandupeux, J. Charmet, R. Bitterli, C. Botta, Y. Rebetez, O. Banakh, H. Keppner, Microelectron. Eng. 2015, 141, 267-279

[2] H. Keppner, M. Benkhaira, WO/2006/063955, 2004

[3] G.-X. Ye, Q.-R. Zhang, C.-M. Feng, H.-L. Ge, Z. Jiao, Phys. Rev. B, 1996, 54, 1475414757

[4] J.-P. Xie, W.-Y. Yu, S.-L. Zhang, M.-G. Chen, G.-X. Ye, Phys. Lett. A, 2007, 371, 160164

[5] E. F. Borra, O. Seddiki, R. Angel, D. Eisenstein, P. Hickson, K. R. Seddon, S. Worden, Nature, 2007, 447, 979-981

[6] N. Binh-Khiem, K. Matsumoto, I. Shimoyama, Langmuir, 2010, 26, 18771-18775

[7] J. Charmet, O. Banakh, E. Laux, B. Graf, F. Dias, A. Dunand, H. Keppner, G. Gorodyska, M. Textor, W. Noell, N. F. de Rooij, A. Neels, M. Dadras, A. Dommann, H. Knapp, Ch. Borter, M. Benkhaira, Thin Solid Films, 2010, 518, 5061-5065

[8] R. J. Frank-Finney, P. D. Haller, M. Gupta, Macromolecules, 2012, 45, 165-170

[9] E. Laux, J. Charmet, H. Haquette, O. Banakh, L. Jeandupeux, B. Graf, H. Keppner, J. Appl. Phys.: Conference Series, 2009, 182, 012029

[10] A. L. Thangawng, R. S. Ruoff, M. A. Swartz, M. R. Glucksberg, Biomed. Microdevices, 2007, 9, 587-595 
[11] A. S. Hoffman, J. Biomat. Sci. - Polym. E. 1999, 10, 1011-1014

[12] P. Wyman, Coatings for Biomedical Applications, Elsevier, 2012

[13] J. Lee, H. Lee, J. Andrade, Prog. Polym. Sci. 1995, 20, 1043-1079

[14] R. I. Mahato, Biomaterials for Delivery and Targeting of Proteins and Nucleic Acids Chapter 1: Structure, Properties, and Characterization of Polymeric Biomaterials, CRC Press, 2004

[15] P. Wang, K.L. Tan, E.T. Kang, K.G. Neoh, J. Membrane Sci. 2002, 195, 103-114

[16] M. Herrmann, E. Roy, T. Veres, M. Tabrizian, Lab Chip, 2007, 7, 1546

[17] G. M. Harbers, K. Emoto, C. Greef, S. W. Metzger, H. N. Woodward, J. J. Mascali, D. W. Grainger, M. J. Lochhead, Chem. Mater. 2007, 19, 4405-4414

[18] K. Fujimoto, H. Inoue, Y. Ikada, J. Biomed. Mater. Res. 1993, 27, 1559-1567

[19] J. H. Lee, B. J. Jeong, H. B. Lee, J. Biomed. Mater. Res. 1997, 34, 105-114

[20] A. Choukourov, I. Gordeev, O. Polonskyi, A. Artemenko, L. Hanyková, I. Krakovský, O. Kylián, D. Slavínská, H. Biederman, Plasma Process. Polym. 2010, 7, 445-458

[21] E. T. Kang, K. G. Neoh, Encyclopedia of Polymer Science and Technology, Wiley, 2009

[22] D. Hegemann, B. Nisol, S. Gaiser, S. Watson, M. R. Wertheimer, Phys. Chem. Chem. Phys. 2019, 21, 8698-8708

[23] J.-H. Han, J.-Y. Yoon, J. Biol. Eng. 2009, 3:6

[24] B. Dong, H. Jiang, S. Manolache, A. C. Lee Wong, F. S. Denes, Langmuir, 2007, 23, 7306-7313

[25] D. Hegemann, I. Indutnyi, L. Zajíčková, E. Makhneva, Z. Farka, Y. Ushenin, M. Vandenbossche, Plasma Process. Polym. 2018, 15, e1800090

[26] B. Dong, S. Manolache, E. B. Somers, A. C. Lee Wong, F. S. Denes, J. Appl. Polym. Sci. 2005, 97, 485-497

[27] A. K. Kenworthy, K. Hristova, D. Needham, T. J. McIntosh, Biophys. J. 1995, 68, 19211936 